\title{
Inhalational drug delivery from seven different spacer devices
}

\author{
Peter W Barry, Christopher O’Callaghan
}

\begin{abstract}
Background - A study was performed to determine in vitro the difference in drug output of seven currently available spacer devices when used with different inhaled medications.
\end{abstract}

Methods - A glass multistage liquid impinger (MSLI) was used to determine the amount of disodium cromoglycate (DSCG, $5 \mathrm{mg})$, salbutamol (100 $\mu \mathrm{g})$, or budesonide $(200 \mu \mathrm{g})$ obtained in various particle size ranges from metered dose inhalers (MDIs) actuated directly into the MSLI or via one of seven different spacer devices: the Fisonair, Nebuhaler, Volumatic, Inspirease, Aerochamber, Aerosol Cloud Enhancer, and Dynahaler.

Results - In particles smaller than $5 \mu \mathrm{m}$ in diameter the dose of DSCG recovered from the Fisonair and Nebuhaler was $118 \%$ and $124 \%$, respectively, of that recovered using the MDI alone. The dose recovered from the smaller volume spacers was $90 \%$ (Inspirease), $36 \%$ (Aerochamber), $33 \%$ (Aerosol Cloud Enhancer), and $21 \%$ (Dynahaler) of that from the MDI alone. The Volumatic increased the amount of salbutamol in particles smaller than $5 \mu \mathrm{m}$ to $117 \%$ of that from the MDI, and the Inspirease and Aerochamber spacers decreased it by nearly $50 \%$. The amount of budesonide in small particles recovered after use of the Nebuhaler, Inspirease, and the Aerochamber was $92 \%, 101 \%$, and $78 \%$, respectively, of that from the MDI alone.

Conclusions - Under the test conditions used, large volume spacers such as the Fisonair, Nebuhaler, and Volumatic delivered significantly more DSCG and salbutamol than the smaller spacers tested. The differences between spacers were less for budesonide than the other medications studied. This study shows that there are significant differences in the amount of drug available for inhalation when different spacers are used as inhalational aids with different drugs. Spacer devices need to be fully evaluated for each drug prescribed for them.

(Thorax 1996;51:835-840)

Keywords: asthma, drug administration, inhalation, spacer devices.

Metered dose inhalers (MDI) are a convenient way of administering medication to patients with asthma and chronic obstructive airways disease. They discharge an aerosol at high speed which is inhaled by the patient. Proper use requires coordination of inhalation and MDI actuation but, even with optimum technique, less than $15 \%$ of the actuated dose reaches the lungs. Spacer devices are intended to improve the efficacy of inhaled therapy by reducing the need for coordination of MDI actuation and inhalation (acting as "holding chambers" for aerosol), and by allowing deceleration and evaporation of drug particles, decreasing extrathoracic deposition of medication (acting as "spacing devices"). They allow effective drug delivery to patients of all ages. ${ }^{1}$

Many different devices are currently available for use in inhalational therapy. Some are intended for use with one particular product, others are marketed as being suitable for use with a variety of MDIs. Inhalation needs to be commenced before MDI actuation with "spacing devices", but not for those designed primarily as "holding chambers". There has been little published work on the comparative efficacy of different devices, and no standard methods for assessing new devices which may be marketed. Metered dose inhalers may contain different formulations with different particle size and aerosol cloud characteristics. Patients vary in size and ability to clear the spacer of medication in a single breath. Factors such as these may alter the combination of MDI, spacer, and patient required for optimum drug delivery and the ideal device for different patients and medications is not known.

We used an impaction particle sizing method to determine the amount of disodium cromoglycate (DSCG), salbutamol, and budesonide available for inhalation from different spacer devices.

\section{Methods}

A glass multistage liquid impinger (MSLI), as modified by Bell et $a l^{2}{ }^{2}$ was used to determine the MDI output under different conditions. The MSLI operates by drawing an aerosol at a constant flow of $60 \mathrm{l} / \mathrm{min}$ through a series of four stages, each containing a glass impaction plate. Aerosol velocity increases progressively through the device and progressively smaller particles collect at each stage. A filter after the final stage collects particles smaller than $1 \mu \mathrm{m}$ in diameter. For DSCG, each stage of the MSLI was washed quantitatively with water. The amount of drug collected in each stage was assayed by ultraviolet spectrophotometry at a wavelength of $326 \mathrm{~nm}$. For salbutamol and budesonide, each stage was washed with 
Table 1 Drug/spacer combinations evaluated

\begin{tabular}{ll}
\hline Drug & Spacer \\
\hline Sodium cromoglycate & Fisonair \\
& Nebuhaler \\
& Inspirease \\
& Aerochamber \\
& Aerosol Cloud Enhancer \\
& Dynahaler \\
Salbutamol & Volumatic \\
& Inspirease \\
& Aerochamber \\
& Dynahaler \\
Budesonide & Nebuhaler \\
& Inspirease \\
& Aerochamber \\
\hline
\end{tabular}

methanol or ethanol, respectively, and the amount of drug collected at each stage determined by high pressure liquid chromatography.

The experiments were undertaken using DSCG, $5 \mathrm{mg}$ per actuation (Intal, Fisons, Loughborough, UK), salbutamol, $100 \mu \mathrm{g}$ per actuation (Ventolin, Allen \& Hanburys, Uxbridge, UK), and budesonide, $200 \mu \mathrm{g}$ per actuation (Pulmicort, Astra Pharmaceuticals, Kings Langley, UK) in conjunction with the following spacers: the Fisonair (Fisons), Nebuhaler (Astra Pharmaceuticals), Volumatic (Allen \& Hanburys), Inspirease (Key Pharmaceuticals, Miami, Florida, USA), Child Aerochamber (Trudell Medical, London, Canada), Aerosol Cloud Enhancer (DHD Corp, Canestota, NY, USA), and the Dynahaler (Healthscan Products, Cedar Grove, NJ, USA). The combinations of drugs and spacers evaluated are given in table 1 . These combinations were chosen as they have been advertised as being appropriate or have been seen in our clinical practice. The features of each device are given in table 2 .

New MDIs of each drug were obtained. The first 10 actuations from each MDI were fired to waste. Immediately before each experiment the MDI was shaken for 30 seconds and primed by firing one actuation. The MDI was then shaken for 10 seconds and actuated into the spacer which was immediately attached to the MSLI. This procedure was repeated 10 times during each experiment to facilitate the drug assay.

Patients using the Aerosol Cloud Enhancer and the Dynahaler are instructed to commence inhalation before MDI actuation. These spacers and, for comparison, the Fisonair were also assessed by connecting the spacer to the MSLI before MDI actuation, mimicking an inspiratory flow through the spacer during actuation of the MDI. These experiments were undertaken with DSCG only.

Table 2 Characteristics of the different spacer devices

\begin{tabular}{|c|c|c|c|c|c|}
\hline Name & Shape & $\begin{array}{l}\text { Length } \\
\text { (cm) }\end{array}$ & $\begin{array}{l}\text { Volume } \\
(\mathrm{ml})\end{array}$ & $\begin{array}{l}\text { Expiratory } \\
\text { valve }\end{array}$ & Comments \\
\hline Nebuhaler & Pear & 23 & 750 & $\checkmark$ & \\
\hline Fisonair & Diamond & 19 & 800 & $v$ & \\
\hline Volumatic & Diamond & 23 & 700 & $\checkmark$ & \\
\hline Inspirease & Cylindrical & 11.3 & 650 & $x$ & $\begin{array}{l}\text { Inspiratory alarm/ } \\
\text { collapsible }\end{array}$ \\
\hline Aerochamber & Cylindrical & 11 & 145 & $v$ & \\
\hline $\begin{array}{l}\text { Aerosol Cloud } \\
\text { Enhancer }\end{array}$ & Pear & 17.5 & 160 & $v$ & Inspiratory alarm \\
\hline Dynahaler & Cylindrical & 12 & 60 & $x$ & \\
\hline
\end{tabular}

Four different Fisonairs, Nebuhalers, Aerosol Cloud Enhancers, Dynahalers, and Volumatic spacers, three Aerochambers, and one Inspirease were used. Experiments were repeated four times with each spacer type. Spacers were all cleaned with water and allowed to dry in air on the laboratory bench before each experiment. The laboratory temperature and relative humidity were recorded for each experiment.

To ensure that the MDI was delivering the prescribed amount of drug under the test conditions it was weighed before and after each experiment. The MDI from each experiment was actuated once into a flask. This was then washed out with the appropriate solvent and the amount of drug released in that actuation determined by analysing the flask washings.

STATISTICAL METHODS

The size distribution of the aerosol cloud was determined from the amount of drug recovered from each stage, the MSLI having been previously calibrated with an aerosol of di-nbutyl phthalate, a non-volatile oil, of known particle size distribution. From this information a log-probability plot was constructed ${ }^{3}$ for each experiment. A line of best fit was derived from this and used to determine the characteristics of the aerosol - for example, the amount of drug contained in particles less than $5 \mu \mathrm{m}$ or $3 \mu \mathrm{m}$ diameter, the mass median aerodynamic diameter (MMAD - the aerodynamic size of a particle such that half of the drug is in larger and half in smaller particles), and the geometric standard deviation (GSD), a measure of the heterogeneity of the aerosol particle size. Mean drug recovery for different devices was compared using analysis of variance (ANOVA). Where a significant difference was detected by ANOVA, recovery between individual devices was compared using unpaired $t$ tests and $\mathrm{p}$ values are given without correction for multiple comparisons.

\section{Results}

Particles smaller than $5 \mu \mathrm{m}$ in diameter are thought to be of optimal size to deliver drug to the lower respiratory tract ${ }^{4}$ and, for simplicity, we refer to this as the "fine particle dose". The amount of drug recovered in particles smaller than $5 \mu \mathrm{m}$ and $3 \mu \mathrm{m}$ in diameter, the MMAD, and GSD were recorded. These results, expressed as the mean and $95 \%$ confidence intervals for each group of experiments, are given in tables 3-6 and, as a percentage of the nominal dose from the MDI, in figs 1-4.

DISODIUM CROMOGLYCATE

There was a significant difference in the fine particle dose delivered from the different devices ( $p<0.00001$, ANOVA; table 3, fig 1). The Fisonair and Nebuhaler increased the fine particle dose by $18 \%$ and $24 \%$ of the MDI dose, respectively $(\mathrm{p}<0.01$ and $\mathrm{p}=0.13, t$ test). The Inspirease delivered $90 \%$ of the MDI fine particle dose whereas the smaller spacers all reduced the fine particle dose to $36 \%$ of that from the MDI with the Aerochamber, 33\% with the Aerosol Cloud Enhancer, 
Table 3 Effect of the use of different spacer devices on the mean amount of sodium cromoglycate recovered (mg) per $5 \mathrm{mg}$ actuation ( $95 \%$ confidence intervals)

\begin{tabular}{llllll}
\hline Method of delivery & Dose to patient & In particles $<5 \mu m$ & In particles $<3 \mu m$ & $M M A D(\mu m)$ & $G S D$ \\
\hline $\begin{array}{l}\text { Direct from metered } \\
\text { dose inhalers }\end{array}$ & $4.14(3.99$ to 4.29$)$ & $0.42(0.40$ to 0.44$)$ & $0.18(0.15$ to 0.20$)$ & $13.8(13.2$ to 14.4$)$ & $2.0(1.8$ to 2.2$)$ \\
Via Fisonair & $1.34(1.25$ to 1.44$)$ & $0.50(0.45$ to 0.54$)$ & $0.24(0.20$ to 0.27$)$ & $6.1(5.9$ to 6.4$)$ & $2.1(2.0$ to 2.2$)$ \\
Via Nebuhaler & $1.2(1.0$ to 1.3$)$ & $0.52(0.42$ to 0.61$)$ & $0.25(0.21$ to 0.28$)$ & $4.9(4.6$ to 5.3$)$ & $2.0(1.98$ to 2.05$)$ \\
Via Inspirease & $0.83(0.75$ to 0.92$)$ & $0.38(0.36$ to 0.40$)$ & $0.2(0.19$ to 0.21$)$ & $5.5(5.1$ to 5.8$)$ & $2.3(2.2$ to 2.3$)$ \\
Via Aerochamber & $0.35(0.22$ to 0.48$)$ & $0.15(0.09$ to 0.20$)$ & $0.07(0.05$ to 0.10$)$ & $5.9(5.6$ to 6.2$)$ & $2.4(2.2$ to 2.6$)$ \\
Via ACE spacer & $0.46(0.40$ to 0.53$)$ & $0.14(0.11$ to 0.17$)$ & $0.07(0.05$ to 0.08$)$ & $10.1(8.3$ to 11.9$)$ & $3.1(2.7$ to 3.5$)$ \\
Via Dynahaler & $0.32(0.21$ to 0.42$)$ & $0.09(0.06$ to 0.12$)$ & $0.04(0.03$ to 0.06$)$ & $8.3(7.7$ to 8.8$)$ & $2.4(2.3$ to 2.4$)$ \\
\hline
\end{tabular}

MMAD = mass median aerodynamic diameter; GSD = geometric standard deviation; ACE = Aerosol Cloud Enhancer.

Table 4 Effect of the use of different spacer devices on the mean amount of sodium cromoglycate recovered ( $\mathrm{mg}$ ) per $5 \mathrm{mg}$ actuation (95\% confidence intervals), sampling during MDI actuation

\begin{tabular}{|c|c|c|c|c|c|}
\hline Method of delivery & Dose to patient & In particles $<5 \mu \mathrm{m}$ & In particles $<3 \mu m$ & $M M A D(\mu m)$ & $G S D$ \\
\hline Via Fisonair & 1.7 (1.50 to 1.89$)$ & $0.50(0.40$ to 0.61$)$ & 0.25 (0.17 to 0.33$)$ & 7.5 (6.8 to 8.2$)$ & $2.2(2.1$ to 2.3$)$ \\
\hline $\begin{array}{l}\text { Via Aerosol Cloud } \\
\text { Enhancer }\end{array}$ & 2.55 (2.48 to 2.62 ) & $0.31(0.24$ to 0.37$)$ & $0.14(0.11$ to 0.17$)$ & 16.55 (12.5 to 20.6$)$ & $2.7(2.1$ to 3.2$)$ \\
\hline Via Dynahaler & $1.0 \quad(0.73$ to 1.27$)$ & $0.26(0.24$ to 0.28$)$ & $0.13(0.12$ to 0.14$)$ & 9.1 (7.4 to 10.7$)$ & 2.5 (2.4 to 2.7$)$ \\
\hline
\end{tabular}

MMAD = mass median aerodynamic diameter; GSD = geometric standard deviation.

Table 5 Effect of the use of different spacer devices on the mean amount of salbutamol recovered ( $\mu \mathrm{g})$ per $100 \mu \mathrm{g}$ actuation (95\% confidence intervals)

\begin{tabular}{llllll}
\hline Method of delivery & Dose to patient & In particles < $5 \mu m$ & In particles < $3 \mu m$ & $M M A D(\mu m)$ & $G S D$ \\
\hline $\begin{array}{l}\text { Direct from metered } \\
\quad 90.5(81.4 \text { to } 99.6)\end{array}$ & $46.3(36.3$ to 56.3$)$ & $34.4(27.5$ to 41.3$)$ & $5.2(3.9$ to 6.5$)$ & $4.8(3.2$ to 6.4$)$ \\
Via Volumate & & & & & \\
Via Inspirease & $62.1(56.0$ to 68.2$)$ & $54.3(48.3$ to 60.1$)$ & $44.9(39.0$ to 50.8$)$ & $1.7(1.6$ to 1.9$)$ & $2.4(2.3$ to 2.5$)$ \\
Via Aerochamber & $30.1(25.2$ to 34.9$)$ & $25.6(21.5$ to 29.7$)$ & $21.0(17.6$ to 24.4$)$ & $1.9(1.7$ to 2.0$)$ & $2.6(2.5$ to 2.6$)$ \\
Via Dynahaler & $23.4(16.3$ to 30.6$)$ & $20.0(15.6$ to 24.5$)$ & $16.2(12.7$ to 19.7$)$ & $2.0(1.2$ to 2.8$)$ & $1.9(1.9$ to 2.3$)$ \\
\hline
\end{tabular}

MMAD = mass median aerodynamic diameter; GSD = geometric standard deviation.

Table 6 Effect of the use of different spacer devices on the mean amount of budesonide recovered ( $\mu \mathrm{g})$ per $200 \mu \mathrm{g}$ actuation (95\% confidence intervals)

\begin{tabular}{llllll}
\hline Method of delivery & Dose to patient & In particles $<5 \mu m$ & In particles < $3 \mu m$ & $M M A D(\mu m)$ & $G S D$ \\
\hline $\begin{array}{l}\text { Direct from metered } \\
\text { dose inhalers }\end{array}$ & $136.5(131.6$ to 141.5$)$ & $33.0(25.5$ to 40.4$)$ & $15.1(12.2$ to 18.0$)$ & $10.2(7.4$ to 13.0$)$ & $2.6(2.3$ to 2.9$)$ \\
Via Nebuhaler & $47.5(38.2$ to 56.8$)$ & $30.5(21.9$ to 39.2$)$ & $15.4(6.7$ to 24.2$)$ & $4.0(3.4$ to 4.7$)$ & $1.8(1.6$ to 2.0$)$ \\
Via Inspirease & $61.3(53.8$ to 68.8$)$ & $33.4(29.8$ to 37.1$)$ & $14.2(12.5$ to 15.9$)$ & $4.7(4.2$ to 5.2$)$ & $1.8(1.7$ to 1.8$)$ \\
Via Aerochamber & $40.2(30.2$ to 50.4$)$ & $25.6(19.5$ to 31.7$)$ & $13.7(11.0$ to 16.4$)$ & $3.9(3.6$ to 4.2$)$ & $2.0(1.8$ to 2.2$)$ \\
\hline
\end{tabular}

MMAD = mass median aerodynamic diameter; GSD = geometric standard deviation .

and $21 \%$ with the Dynahaler. There was no difference between the fine particle dose delivered from either of the two larger volume spacers - the Nebuhaler and the Fisonair - or between the smaller volume spacers - the Aerochamber, Aerosol Cloud Enhancer, and the Dynahaler. All the spacers except the Aerosol Cloud Enhancer reduced the MMAD of the aerosol compared with the MDI used without a spacer. This effect was greatest for the larger volume spacers, and for the Nebuhaler compared with the Fisonair $(p<0.01)$.

Sampling during MDI actuation, to represent coordination of inhalation and MDI actuation into the spacer, increased recovery of DSCG from the Aerosol Cloud Enhancer (0.14 to $0.31 \mathrm{mg})$ and Dynahaler $(0.09$ to 0.26 mg) (tables 3 and 4, fig 2). However, these spacers delivered less drug in small particles than the MDI or Fisonair assessed under the same conditions ( $p<0.001$, ANOVA).
SALBUTAMOL

There was a significant difference in the fine particle dose delivered from the different devices (table 5, fig 3; $\mathrm{p}<0.00001$, ANOVA). The Volumatic increased the fine particle dose by $17 \%(p=0.05)$, and the smaller spacers reduced the fine particle dose compared with the MDI alone to $55 \%$ with the Inspirease and $43 \%$ with the Aerochamber. So little drug was obtained from the Dynahaler that a particle size distribution was impossible to obtain. With this exception, the spacers all equally reduced the MMAD of the aerosol compared with the MDI used without a spacer.

\section{BUDESONIDE}

There was no statistically significant difference between the different spacers in the delivery of budesonide (table 6, fig 4). The Nebuhaler and the Aerochamber reduced the fine particle 


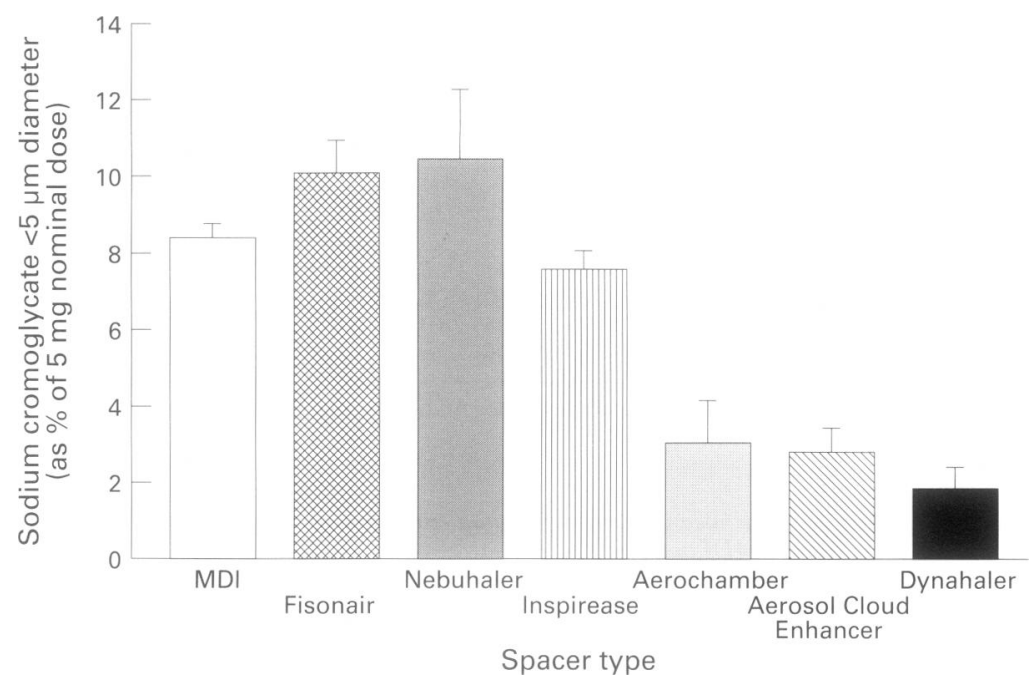

Figure 1 Amount of sodium cromoglycate (percentage of $5 \mathrm{mg}$ dose) recovered in particles smaller than $5 \mu \mathrm{m}$ aerodynamic diameter for seven different spacer devices. Vertical bars represent one standard deviation.

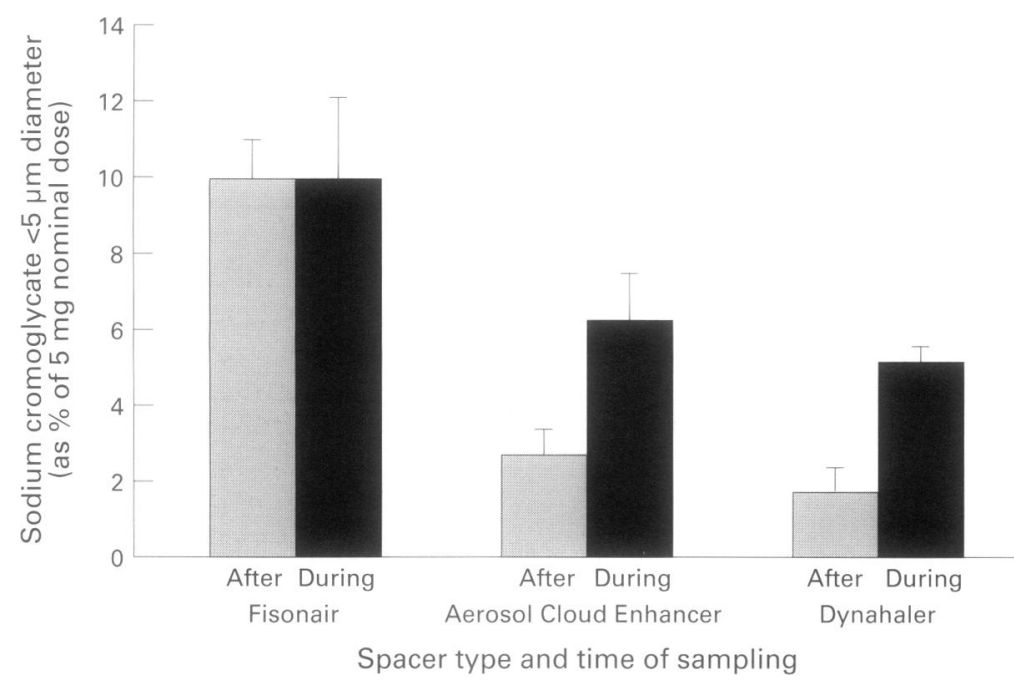

Figure 2 Amount of sodium cromoglycate (percentage of $5 \mathrm{mg}$ dose) recovered in particles smaller than $5 \mu \mathrm{m}$ aerodynamic diameter for three spacer devices sampled either one second after (shaded bars) or during (black bars) metered dose inhaler actuation.

dose to $92 \%$ and $78 \%$, respectively, compared ith the MDI whereas the Inspirease delivered the same amount of drug as the MDI. Reducing extrathoracic drug deposition is an important effect of spacer devices used with inhaled steroids and the three spacers all reduced the delivery of larger particles. Again the spacers all equally reduced the MMAD of the aerosol

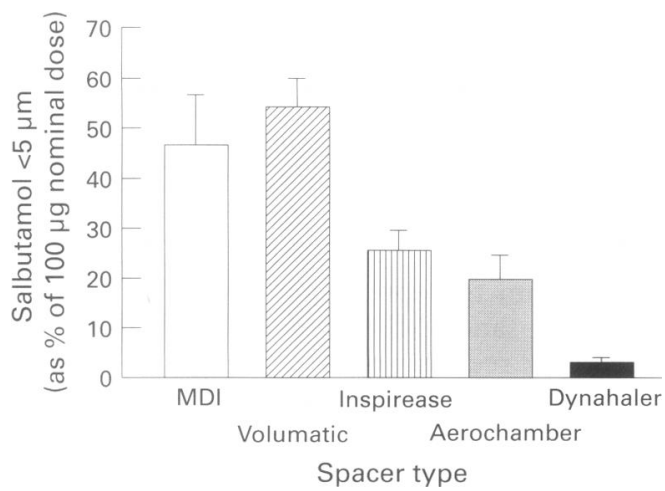

Figure 3 Amount of salbutamol (percentage of $100 \mu \mathrm{g}$ dose) recovered in particles smaller than $5 \mu \mathrm{m}$ aerodynamic diameter for five different spacer devices.

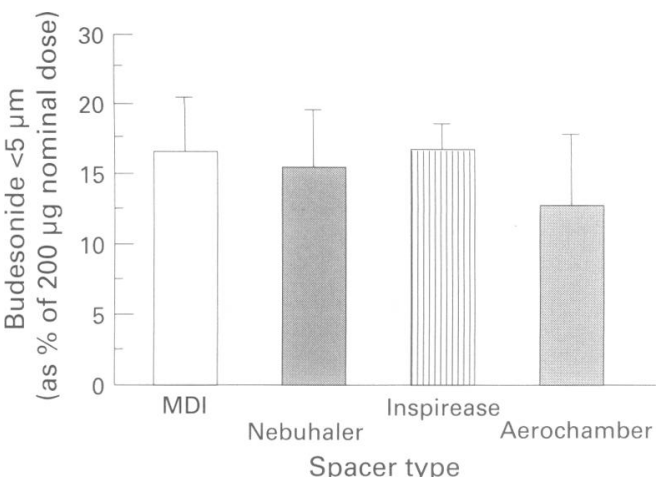

Figure 4 Amount of budesonide (percentage of $200 \mu \mathrm{g}$ dose) recovered in particles smaller than $5 \mu \mathrm{m}$ aerodynamic diameter for three different spacer devices.

compared with the MDI used without a spacer and the total drug delivery was a quarter of that from the MDI when a spacer was used $(\mathrm{p}<0.0005$, ANOVA)

The mean (SD) amount of drug recovered after a single actuation into a flask was 4.36 (0.28) $\mathrm{mg}$ for DSCG, 101 (13) $\mu \mathrm{g}$ for salbutamol, and 184 (9.5) $\mu \mathrm{g}$ for budesonide. Mean (SD) MDI weight loss per actuation was DSCG 138 (4.5) mg, salbutamol 86 (1.2) mg, budesonide 73 (2.6) mg. Coefficient of variation of the drug assay was $0.2 \%$ for DSCG, $2.1 \%$ for salbutamol, and $6 \%$ for budesonide.

During the experimental period the mean temperature was $23^{\circ} \mathrm{C}$ (range $21-25$ ) and the mean relative humidity was $55 \%$ (range 51-63).

\section{Discussion}

This study demonstrates large variations in the amount of drug delivered from different spacer devices, and variations in the relative efficacy of a spacer to deliver different drugs. The Aerochamber, for instance, delivers only $36 \%$ of the fine particle dose of DSCG obtained when the MDI is used without a spacer, but $78 \%$ of the equivalent dose from the budesonide MDI. Both the Aerosol Cloud Enhancer and the Dynahaler delivered very small amounts of medication (less than $33 \%$ of the MDI fine particle dose). It is clear from these results that experiments with one spacer device or drug cannot be extrapolated to others.

Others have examined the effect of different size and shaped spacer devices on drug delivery in vitro, but have not investigated the particle size distribution of the spacer output, one of the main determinants of the site of drug deposition. Moren ${ }^{5}$ measured oral and spacer deposition of terbutaline using spacers of different length, diameter and shape and found that increasing the spacer length from $5 \mathrm{~cm}$ to $10 \mathrm{~cm}$ decreased oral deposition of drug but did not affect total drug recovery or, by inference, drug delivery to the patient. Maximum drug delivery was achieved using a pear shaped spacer of $25 \mathrm{~cm}$ length.

Kim et al compared the in vitro delivery of a variety of inhaled steroids and bronchodilators used with the Nebuhaler, Aerochamber, and Inspirease. They found that the large volume Nebuhaler substantially increased the aerosol delivery to the lung of their model (by up to 
$38 \%$ compared with the MDI), but that the smaller Aerochamber and Inspirease did not. They found increased delivery to their model lung when steroid suspensions were compared with bronchodilator solutions delivered through the smaller spacers, similar to our finding of less difference between spacers when used with the budesonide MDI.

Patient size and breathing pattern may also be important in the choice of spacer device. Particles settle in the spacer after only a few seconds ${ }^{78}$ and, from our results, the concentration of fine particles in small spacers is higher than in larger ones. This may be important in small children, for whom a few small breaths from concentrated aerosol in a small spacer may deliver more drug than would be obtained from a large spacer. This is supported by an in vitro study evaluating the total dose of DSCG aerosol obtained at different tidal volumes through spacer devices ${ }^{9}$ which showed that the Aerochamber delivered more drug than the Nebuhaler at tidal volumes below $50 \mathrm{ml}$, but that this was reversed at a tidal volume of $150 \mathrm{ml}$.

Other factors such as static charge, ${ }^{7}$ spacer shape, or valve design ${ }^{10}$ may be involved in determining drug delivery. These and other important factors in spacer design and use have not been investigated adequately.

In radiolabelled and clinical studies of adults and older children, large cone spacers are more effective than the MDI used alone ${ }^{111} 12$ whereas small volume spacers are only as effective as the optimally used MDI. ${ }^{13}{ }^{14}$ Both the small and large volume spacers reduce oropharyngeal deposition and side effects of inhaled steroid therapy. ${ }^{15}$ There are few clinical data comparing different spacers in the delivery of inhaled steroids. Differences in clinical response to bronchodilators delivered via different spacer devices have been described in some studies, ${ }^{16-18}$ but not all, ${ }^{19}{ }^{20}$ perhaps because the dose of drug delivered tends to fall on the flat part of the dose response curve..$^{21}$ The clinical relevance of small differences in bronchodilation with different spacers is not clear.

Although some spacers are physically suitable for many different brands of MDI and have been recommended for a range of inhalers, ${ }^{22}$ it may not be possible to use the results of an experiment with one particular MDI and spacer to predict the performance of the spacer with another drug. We have shown that differences in the delivery of budesonide from different spacers are not as great as those observed with DSCG and salbutamol. This may be due to a number of factors, such as decreased aerosol density with budesonide (one actuation of budesonide weighs 60 $70 \mathrm{mg}$ compared with $140 \mathrm{mg}$ for DSCG), differences in aerosol cloud morphology, or interaction with the spacer walls due to static charge. The imminent phasing out of chlorofluorocarbon use in MDIs may change the aerosol cloud characteristics and necessitate a re-evaluation of MDIs and spacer devices.

Both the Dynahaler and Aerosol Cloud Enhancer gave very poor output of respirable drug. However, these spacers are designed for adults who will be able to commence inhalation just before MDI actuation. Even when sampling is commenced before MDI actuation, mimicking the optimum clinical use, the spacers deliver small amounts of DSCG relative to a large volume spacer or the MDI alone. Up to $70 \%$ of adults are not able to coordinate the hand-breath manoeuvres of MDI use. ${ }^{21}$ Our study implies that the Dynahaler and Aerosol Cloud Enhancer may not be as useful for patients with poor inhaler technique.

All the spacers tested reduced the total amount of drug delivered from the spacer compared with the MDI alone and, in most cases, reduced the size of drug particles that were delivered, shown by the reduction in MMAD of the aerosol from the spacers. This is one advantage of spacer devices, as larger drug particles are retained in the device rather than being deposited in the oropharynx where they may contribute to drug related side effects.

The MSLI uses a series of inertial impactors to fractionate the aerosol cloud by particle aerodynamic size. It samples at a fixed flow rate of $601 / \mathrm{min}$ and is not intended to mimic respiration but to determine particle size, one of the main determinants of aerosol deposition. The issues of inspiratory flow, airway structure, and airway calibre are not addressed in this study in which we have determined the amount of drug below a certain size that is available for inhalation. The choice of $5 \mu \mathrm{m}$ to define the "fine particle dose" is also arbitrary as the optimum particle size for an inhaled medication will depend on many factors such as inspiratory flow, upper airway geometry, and airway calibre. The MSLI may underestimate particle size due to desiccation of particles as they pass through the system, in contrast to the respiratory tract where hygroscopic drug particles would be expected to grow significantly in size. ${ }^{23}$ This would affect all the spacers in the study, however, allowing devices to be ranked in terms of their drug output and valid comparisons between devices to be made.

We have shown that the in vitro delivery of drug in small particles varies greatly between different spacer devices and that the efficacy of a particular spacer with one drug cannot be assumed for another drug. The factors underlying this and the design of the optimum spacer are yet to be determined and confirmed in clinical trials, but our data suggest that it is inappropriate to use any drug with any device uncritically just because the MDI adapter fits.

Dr Barry is funded by the Astra Foundation. We thank Mrs J Jackson for technical help, Fisons plc, Allen \& Hanburys Ltd, and Astra Ltd for supplying the metered dose inhalers, the various companies for supplying their spacer devices, and Astra Draco, Fisons plc and Glaxo Group Research for technical advice on the drug assays.

1 Levison H, Reilly PA, Worsley GH. Spacing devices and metered dose inhalers in childhood asthma. F Pediatr 1985; 107:662-8.

2 Bell JH, Brown K, Glasby J. Variations in the delivery of isoprenaline from various pressurised inhalers. 7 Pharm Pharmacol 1973;25(Suppl):32-6.

3 Hinds WC. Aerosol technology. New York: John Wiley \& Sons, 1983.

4 Stalhofen W, Gebbert J, Heyder J. Experimental determination of the regional deposition of aerosol particles in the human respiratory tract. Am Ind Hyg Assoc $尹 1980 ; 41$ :38599 
5 Moren F. Drug deposition of pressurised inhalation aerosols 1. Influence of actuator tube design. Int $\mathcal{f}$ Pharm 1978;1:205-12.

6 Kim CS, Eldridge MA, Sackner MA. Oropharyngeal deposition and delivery aspects of metered dose inhaler aerosols. Am Rev Respir Dis 1987;135:157-64.

7 O'Callaghan C, Lynch J, Cant M, Robertson C. Improvement in drug delivery from spacer devices by use of an antistatic lining. Thorax 1993;48:603-6.

8 Barry PW, Robertson C, O'Callaghan C. Optimum use of a spacer device. Arch Dis Child 1993;69:693-4.

9 Everard ML Clark AR, Milner AD. Drug delivery from holding chambers with attached facemask. Arch Dis Child 1992;67:580-5.

10 Sennhauser FH, Sly PD. Pressure flow characteristics of the valve in spacer devices. Arch Dis Child 1989;64:1305-19.

11 Cushley MJ. Comparison of three techniques of inhalation on the airway response to terbutaline. Thorax 1983; 38:908-13.

2 Newman SP. Improvement of pressurised aerosol deposition with the Nebuhaler spacer device. Thorax 1984;39:93541.

13 Dolovich M, Eng P, Ruffin R, Corr D, Newhouse MT. Clinical evaluation of a simple demand inhalation aerosol delivery device. Chest 1983;84:36-41.

14 Gurwitz D, Levison H, Mindorff C, Reilly P, Worsley G. Assessment of a new device (Aerochamber) for use with assosol drugs in asthmatic children. Ann Allergy 1983; 50:166-70.
15 Selroos $\mathrm{O}$, Halme $M$. The effect of a Volumatic spacer and mouth rinsing on systemic absorption of inhaled corticomouth rinsing on systemic absorption of inhaled corticoinhaler. Thorax 1991;46:891-4.

16 Dhand R, Sethi DS. Bronchodilator effect of terbutaline aerosol in asthmatics: comparison of two spacer devices. Indian $f$ Chest Dis Allied Sci 1990;32:101-6.

17 Crimi N, Palermo F, Cacopardo B, Vancheri C, Oliveri R, Palermo B, et al. Bronchodilator effect of Aerochamber and Inspirease in comparison with metered dose inhaler. Eur F Respir Dis 1987;71:153-7.

18 Lee H, Evans HE. Evaluation of inhalation aids of metered dose inhalers in asthmatic children. Chest 1987;91:366-9.

9 Lulling J, Delwiche JP, Hidinger KG, Prignot J. Influence of different extension-actuator tubes on the bronchodilation effect of a terbutaline sulfate aerosol. Eur 7 Respir Dis 1983;64:33-7.

20 Fuller HD. Comparison of two chamber devices in patients using a metered dose inhaler with satisfactory technique. Can Med Assoc ₹ 1986;135:625-9.

21 Sackner MA, Kim CS. Auxiliary MDI delivery systems. Chest 1985;88: 161-70S

22 Hodges IGC, Milner AD, Stokes GM. Assessment of a new device for delivering aerosol drugs to asthmatic children. Arch Dis Child 1981;56:787-9.

23 Hicks JF, Megaw WJ. The growth of ambient aerosols in the conditions of the respiratory system. $f$ Aerosol Sci $1985 ; 16: 521-7$ 